\title{
Performance of Tau Identification and Associated Systematic Uncertainties in ATLAS
}

\section{Frank SEIFERT ${ }^{* \dagger}$}

Institut für Kern- und Teilchenphysik, Technische Universität Dresden, D-01062 Dresden.

E-mail: F.Seifert@physik.tu-dresden.de

\begin{abstract}
In these proceedings, a description of the reconstruction and identification methods for tau leptons in ATLAS at the LHC is presented. Identifying tau leptons is essential in many analyses, be it for Standard Model (SM) and Higgs boson signatures or searches for physics beyond the SM. The decay of the tau particle with leptonic as well as hadronic decay modes and its short lifetime makes it especially challenging to identify this lepton. After a short introduction about the tau lepton and its reconstruction in ATLAS, three different tau identification methods are presented in more detail. The identification efficiencies and their systematic uncertainties for 2010 data analysis in ATLAS are evaluated and discussed here.
\end{abstract}

XXIst International Europhysics Conference on High Energy Physics

21-27 July 2011

Grenoble, Rhône-Alpes France

* Speaker.

$\dagger$ On behalf of the ATLAS Collaboration. 


\section{Introduction}

The tau lepton has a mean lifetime of $2.9 \cdot 10^{-13} \mathrm{~s}$ and is the only lepton which is massive enough to decay hadronically. With $c \tau=87 \mu \mathrm{m}$, it decays already inside the ATLAS [1] beam pipe and the tau particle can only be measured indirectly via its decay products. The branching ratio is $17.9 \%$ into $e v_{e} v_{\tau}, 17.4 \%$ into $\mu v_{\mu} v_{\tau}$ and $64.7 \%$ into hadrons. Since leptonic tau decays are difficult to distinguish from primary electrons or muons, only hadronic tau decays are considered for tau reconstruction and identification in ATLAS.

Measurements of the tau decay products are done with inner detector tracks and calorimeter clusters. Due to their similar decay signature, QCD processes are the most important background in tau reconstruction and identification. Detector variables with discrimination power can be used in cut-based methods or as inputs to multivariate discriminants to distinguish between hadronic tau decays and QCD jets on a statistical basis. Physics analyses must find an optimal balance between high tau identification efficiency and high rejection rate against QCD jets.

\section{Tau Reconstruction}

Tau reconstruction in ATLAS is based on calorimeter jets with a transverse momentum, $p_{T}$, greater than $10 \mathrm{GeV}$, which are used as a seed for the tau reconstruction algorithm. Inner detector tracks with $p_{T}>1 \mathrm{GeV}$ and within a cone of $R=\sqrt{\Delta \eta^{2}+\Delta \varphi^{2}}<0.2$, relative to the seed jet, are associated to the tau candidate. Both, calorimeter and tracking variables are used for the tau reconstruction. The background rejection of the tau reconstruction itself is small and higher rejection rates are provided by the various tau identification methods.

During the reconstruction step, the combined variables, used in the different identification methods, are calculated. A detailed description of these variables can be found in Reference [2]. Only a subset of these variables are used in each identification method.

\section{Cut-based Identification}

The cut-based tau identification method uses simple cuts on the three variables: electromagnetic radius, $R_{\mathrm{EM}}$, track radius, $R_{\text {track }}$, and momentum fraction of the leading track, $f_{\text {track}}$. The cuts on $R_{\mathrm{EM}}$ and $R_{\text {track }}$ are dependent on $p_{\mathrm{T}}$ to take into account the Lorentz boost of the tau decay products in the laboratory frame. Due to this collimation, variables describing the lateral extent of the energy deposition, $R$, depend on $p_{\mathrm{T}}$ as $R\left(p_{\mathrm{T}}\right) \propto \frac{1}{p_{\mathrm{T}}}$. An example of the distribution and $p_{\mathrm{T}}$-dependent cut on $R_{\mathrm{EM}}$ are shown in the left picture of Fig. 1 .

Systematic uncertainties on the identification efficiencies are evaluated in bins of $p_{\mathrm{T}}$, separately for 1-track and multiple-track candidates, and for high or low multiplicity of proton-proton interaction vertices to account for different pile-up scenarios. These uncertainties have been evaluated using different Monte Carlo samples with variations in the event generation, detector material, shower modeling, and reconstruction. 


\section{Multivariate Identification Methods}

One multivariate identification method is the projective likelihood, which uses three discriminating variables for 1-prong tau decays and five for 3-prong tau decays. The likelihood score is based on the product of probability density functions, $p$, for signal, $S$, and background, $B$ :

$$
d=\ln \frac{L_{S}}{L_{B}}=\sum_{i=1}^{N} \ln \frac{p_{i}^{S}\left(x_{i}\right)}{p_{i}^{B}\left(x_{i}\right)}
$$

where $N$ is the number of variables used. Looser and tighter cuts on the likelihood score are defined to obtain identification efficiencies of approximately $60 \%$, and $40 \%$, respectively.

The second multivariate method is the identification with boosted decision trees (BDT) using up to nine variables in a series of cuts. The training is done separately for 1-prong and 3-prong tau candidates, and also for events with 1-2 or more than two primary vertices. The $p_{\mathrm{T}}$-dependent cuts on the BDT score are optimized to provide approx. flat signal or background efficiency.
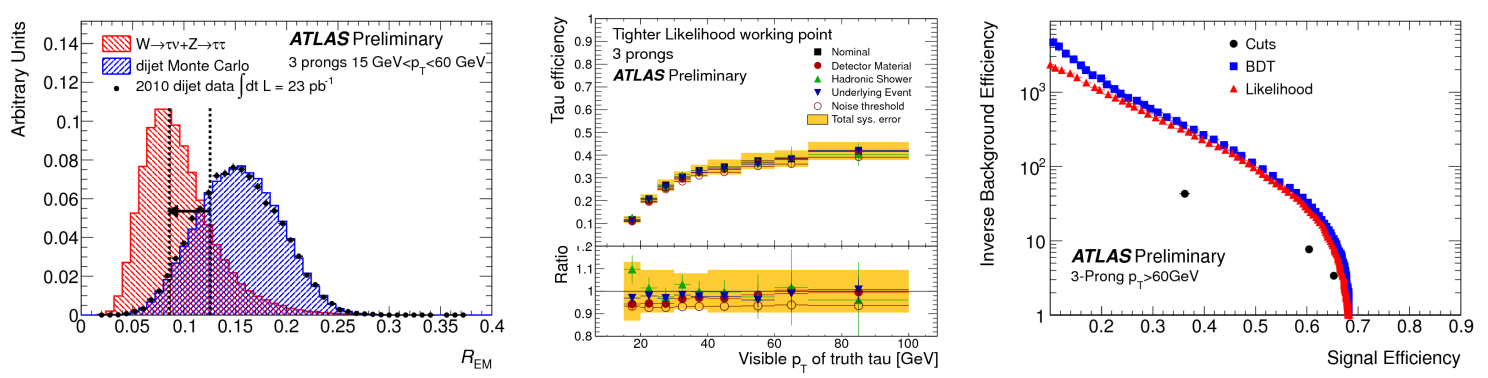

Figure 1: Left: distributions of $R_{\mathrm{EM}}$ for signal Monte Carlo and dijet collision data compared with Monte Carlo predictions for 3-prong tau candidates. The $p_{\mathrm{T}}$-dependent cuts are indicated as vertical lines and the arrows denote the range from $20 \mathrm{GeV}$ to $60 \mathrm{GeV}$. Middle: identification efficiency of a tight working point of the likelihood method with assigned systematic uncertainties. Right: comparison of the overall performance between cut-based, likelihood and BDT identification methods for 3-prong tau candidates [2].

\section{Systematic Uncertainties and Conclusion}

The overall performance of the multivariate methods is improved with respect to the cut-based one as illustrated in the right picture of Fig. 1. The performance presented here is as obtained for the 2010 data analysis. Background rejection factors of more than 1000 at efficiencies of $20 \%$ are possible. The overall systematic uncertainties are in a range between $4 \%$ and $12 \%$, depending on the working point and the and number of tracks and $p_{\mathrm{T}}$ of the tau candidate [2].

\section{References}

[1] The ATLAS Collaboration, G. Aad et al., The ATLAS Experiment at the CERN Large Hadron Collider, JINST 3 S08003, 2008.

[2] The ATLAS Collaboration, Reconstruction, Energy Calibration, and Identification of Hadronically Decaying Tau Leptons in the ATLAS Experiment, ATLAS-CONF-2011-077. 